\title{
A Within-Attribute Model of Variety-Seeking Behavior
}

\author{
MICHAEL D. JOHNSON \\ School of Business Administration, University of Michigan, Ann Arbor, Michigan 49109-1234 \\ ANDREAS HERRMANN \\ University of Mannheim \\ JENS GUTSCHE \\ University of Mannheim
}

Key words: cognitive representation, variety seeking, satiation

\begin{abstract}
Existing models view variety seeking as the result of differences in the level of attribute satiation across attributes. An alternative within-attribute variety-seeking (WAVS) model is proposed. The model posits that variety seeking occurs among the nested features, or meaningful value ranges, of an underlying dimension. The resulting pattern of consumption is represented as an oscillation about a consumer's ideal point on the dimension. An empirical study that illustrates different oscillation patterns is reported.
\end{abstract}

Consumers frequently switch among brands in a category. This brand switching may be labeled variety seeking when, for instance, familiar brands and intrapersonal causes are involved. Recent attribute satiation or balance models offer a compelling description of the dynamics of variety seeking in this context (Bawa, 1990; Jeuland, 1978; Lattin, 1987; McAlister, 1982; Lattin and McAlister, 1985). These models explain variety seeking by way of the level of satiation on desired attributes at a given point in thine. A cominion thivad running through these models is that variety seeking is driven by competition across attributes; when one attribute becomes satiated, consumers switch to a product that contains some other preferred and less satiated attribute.

The goal of this study is to offer a fundamentally different view of consumers' cognitive representations of products and their attributes and illustrate its major prediction. A model is proposed in which variety seeking among familiar brands occurs within the context of a particular attribute. This variety seeking results in a cycling or oscillation around the ideal point on the attribute. After developing the model we describe a procedure for operationalizing the predicted oscillation patterns and provide an empirical illustration involving beverages.

\section{Within-attribute variety seeking}

Of all the possible causes of variation in brand choice, we focus on the inherently satisfying aspects of varied behavior among familiar alternatives (such as beverages and snack 
foods). The prevailing view of variety seeking in this context is that consumers alternate among known brands to balance the weak preference for some brands over others and the boredom or level of satiation created by extended exposure to a particular product or product attribute (Farquhar and Rao, 1976; Givon, 1984; Jeuland, 1978; Lattin, 1987; Lattin and McAlister, 1985; McAlister, 1982). Thus a consumer's satiation on "sweetness," and their resulting preference for a sweet beverage, should depend on their recent consumption history and existing inventory on this dimension. A common theme in existing satiationbased models is that variety seeking is the result of satiation across attributes. When one attribute becomes satiated, preference becomes driven by a different attribute that is less satiated at the time of choice. The model proposed herein differs from existing models in that variety seeking occurs within the setting of a particular attribute.

Research on cognitive representations demonstrates that the attributes or characteristics that consumers use to describe familiar brands are both relatively abstract or summary in nature and inherently dimensional (Johnson and Fornell, 1987; Johnson et al., 1992). These descriptive attributes vary continuously as a matter of degree, such as the level of fruitiness in a beverage or sportiness in a vehicle. Like previous models, we presume that consumers hold some ideal level of satiation on these attributes. Unlike previous models, we presume a second order of preference and satiation such that consumers seek variation within an attribute. This presumption is based on another important finding from the cognitive representation literature: when processing familiar brands, consumers apply the principles of categorization to descriptive dimensions and process them categorically as nested features (Johnson et al., 1992; Park, 1976; Prinz and Scheerer-Neuman, 1974). These features represent meaningful value ranges on the underlying dimension, which may separate products by the type of experience they provide or the acceptability of options (Myers and Shocker, 1981; Park, 1978; Gati and Tversky, 1982).

Viewing descriptive attributes as nested features allows us to posit a distribution of preferences across the levels of an underlying dimension (Best, 1976; Lehmann, 1972). Each meaningful value range, or nested feature, has some independent preference that is distributed more or less symmetrically about the "ideal" or most preferred value range. Each feature also has a relatively independent level of satiation. While beverages vary on fruitiness, customers may distinguish categorically between nonfruity, fruity, and very fruity beverages. While fruity beverages may be most preferred, or where their stated ideal point is located, there may be some independent preference (and satiation) for nonfurity and very fruity beverages. These categories are relatively independent in the sense that no amount of a fruity beverage will completely satiate a weaker preference for a very fruity beverage, while no degree of abstinence from a fruity beverage will satiate a weaker preference for a nonfruity beverage.

The key prediction of the within-attribute variety-seeking (WAVS) model is that consumption of the attribute should oscillate predictably about the ideal point on the dimension. Consider the above example where consumers cognitively represent fruitiness as three distinct value ranges: nonfruity, fruity, and very fruity. Say that the consumer's preference distribution over these value ranges is symmetric about the ideal level of the attribute, which happens to be in the fruity range, such that $p$ (fruity) $>p$ (nonfruity) $=p$ (very fruity) $>0$ where $p$ is the level of preference for the value range. This distribution of preferences suggests that consumers at time period $t_{1}$ will choose a fruity beverage. Given a sufficient 
retention factor, the consumer's weaker preference for a nonfruity beverage might dictate the choice of such a beverage at time $t_{2}$. The resulting inventory decay for fruity beverages may dictate a return to this "ideal" value range when a choice is made at time $t_{3}$. Under this scenario, it is likely that at time $t_{4}$ the consumer will choose a very fruity beverage, their preference for which is unsatiated relative to fruity and nonfruity beverages. Continuation of this logic results in an oscillating pattern of preferences over time about the "ideal." While it is possible that such a prediction may result from across-attribute variety seeking, it is unlikely. It would require a very specific pattern of relationships across the attributes with regard to their levels of satiation and retention as well as symmetry in their relative preference.

The nature of the predicted oscillation depends on several factors. The stronger or more peaked the preference distribution is for the ideal attribute level, or the lower the retention factor, the more the ideal point should drive choice behavior. Such consumers will be relatively "value loyal." The weaker the relative preference for the ideal point, or the higher the retention factor, the larger the magnitude of the oscillations. Naturally, the prediction of oscillating preferences rests on the assumption that preferences are distributed relatively symmetrically about the ideal. This assumption may be more problematic in some contexts than others, especially when the ideal point is bounded by some minimum or maximum level on the dimension resulting in a skewed preference distribution.

\subsection{Estimation procedure}

We describe a procedure for estimating the predicted oscillations of the WAVS model. The information required includes (1) consumer perceptions of the stimuli in question on the underlying attribute of interest (such as perceptions of beverages on fruitiness), (2) consumer perceptions of the ideal point on the attribute of interest, (3) a retention factor, and (4) consumer purchase histories for the brands used to satiate the attribute. Note that only information on the underlying descriptive dimension, not the nested features on this dimension, is required in this procedure.

The influence of the consumption experience at time $t-1$ on the level of the attribute preferred at $t$ is taken into account by multiplying the level of the attribute experienced at $t-1$ by a retention factor. This retention factor specifies the rate at which a consumption experience on the underlying descriptive dimension dwindles over time. If the factor is high (low), the consumption experience at time $t-1$ is very firmly (loosely) anchored in the consumer's memory at time $t$. McAlister (1982) assumed a retention value of .5 in a beverage consumption context, and we adopt this same value as the basis for our empirical study. We later estimate the model using retention values of .25 and .75 for comparison purposes.

The resulting attribute inventory is then added to the perceived amount of the attribute in the product purchased and consumed at $t$. Using the concept of revealed preferences, we presume that this sum reveals the preferred level of consumption on the attribute at time $t$. The deviation of this preferred attribute level from the ideal is then represented as follows (McAlister 1982):

$$
k_{t i d}=\left(I_{t d}+X_{i d}\right)-X_{d}^{*},
$$


where $k_{t i d}$ is the deviation from the consumer's ideal point on attribute $d$ obtained from consuming product $i$ at time $t, X_{d}^{*}$ is the consumer's ideal level of attribute $d, I_{t d}$ is the consumer's inventory of attribute $d$ at time $t$, and $X_{i d}$ is the amount of attribute $d$ in product $i$. The value of $X_{d}^{*}$ represents the ideal intensity of the consumption experience on $d$ over time, while $k_{t i d}$ represents deviation from the consumer's stated, or average, ideal at any given point in time, as revealed by the consumer's choice, in light of their consumption history. Following Jeuland (1978), we define the attribute inventory as

$$
I_{t d}=h \cdot k_{t-1 i d}
$$

where $h$ is the retention factor described above such that $0 \leq h \leq 1$. The higher the value of $h$, the more firmly the consumption experience from the previous period is anchored in the buyer's memory. The next step in the procedure is to group respondents with respect to the similarity in their $k_{t i d}$ values over time, or $k_{t i d}$ cycles, and estimate the oscillation pattern for each segment. These steps are illustrated by way of an empirical study of beverages.

\section{Example}

An empirical study was conducted to illustrate the predicted patterns of oscillation. The study involved a convenience sample of thirty-eight consumers from Koblenz, Germany, who consumed at least one of the brands each day and averaged one such beverage per day. The respondents recorded the beverage they chose to consume on thirty consecutive days from among Lipton's Iced Tea, Coke, Pepsi, Fanta, Sprite, and Rhodius Mineral Water. The respondents were instructed to plan their purchases so that at least one soft drink was consumed every day.

We focused on the attribute "fruitiness," a salient underlying or latent attribute used to describe differences among beverages. At the beginning of the study, each of the consumers was asked to rate their perception of each of the five beverages on fruitiness using a scale from 0 (not at all fruity) to 5 (very fruity) and to specify their ideal level of this attribute. The available data was substituted into equations (1) and (2) to calculate the $k_{t i d}$ values for each respondent for each period.

Note that because the attribute inventory at time $t_{1}$ is always zero (it does not reflect the effect that previous consumption experiences might have on present brand choice), we ignored the first four purchase acts in our estimation of the oscillation patterns; at most only the last twenty-six out of the total of thirty purchase acts were considered. Also, to make the individual $k_{\text {tid }}$ cycles comparable with one another, we determined a common $k_{t i d}$ value for all consumers before beginning the analysis. In our study, the first positive peak of the "sinusoidal" configuration of each test person's $k_{t i d}$ values was defined as time period $t_{1}$. The end result is that the actual number of purchase occasions used in the estimation ranged from a minimum of twenty-one to a maximum of twenty-six across individuals.

Cluster analysis was used to identify segments of consumers with similar oscillation patterns. Correlations between the $k_{\text {tid }}$ values across purchase occasions for each pair of subjects were used as inputs to Ward's agglomerative-hierarchical method, which revealed four clusters or types of respondents who were similar in their oscillation cycles. The $k_{t i d}$ values for the customer types are presented in Figure 1 (the numbers 1, 2, 3, and so on in the 


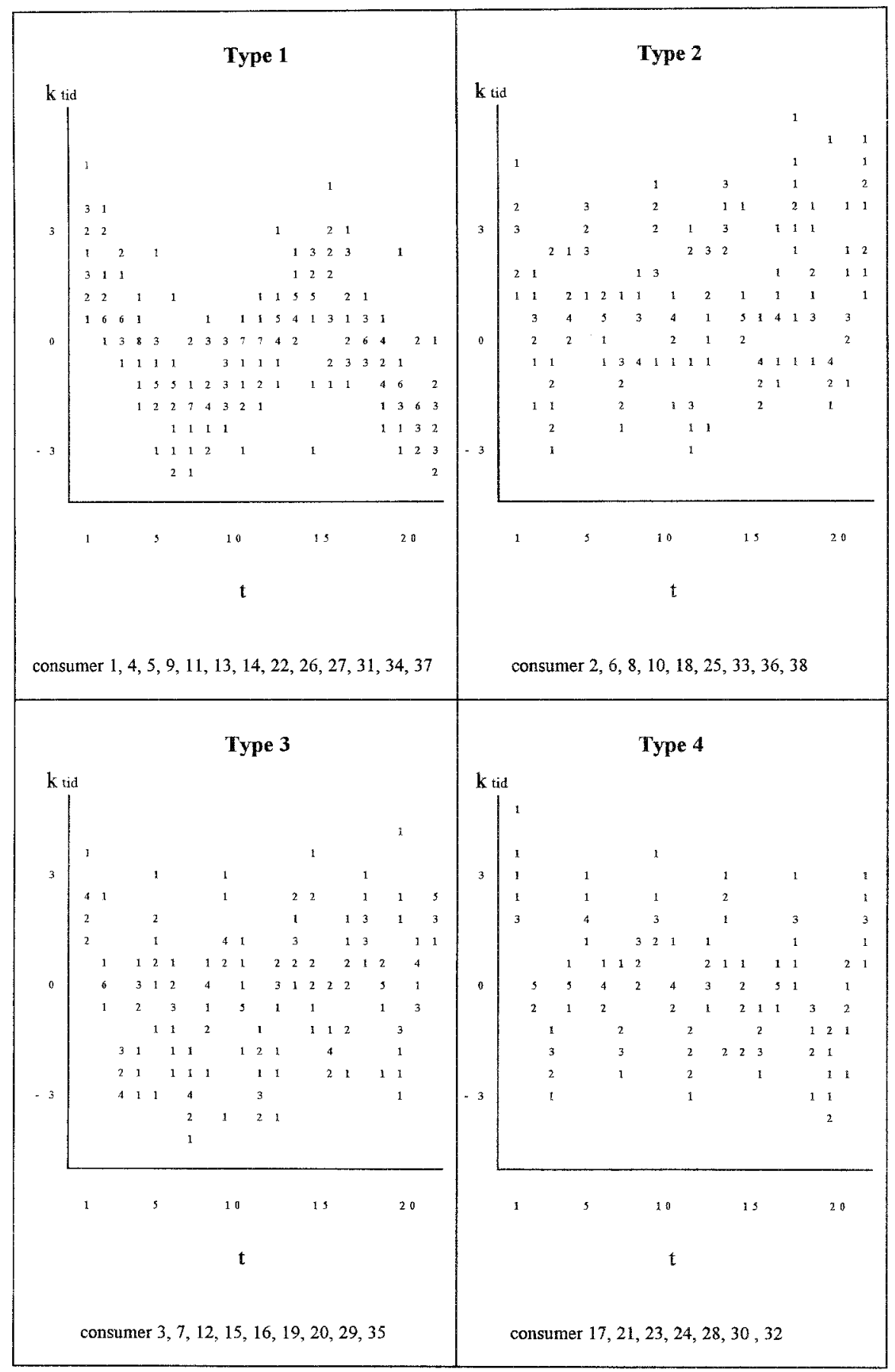

Figure 1. Value for $k_{t i d}$ over time by consumer type. 
graphs represent the number of observations). Type 1 consumers $(n=13)$ have $k_{t i d}$ values that are relatively stable and oscillate about zero by a significant amount. Their $k_{\text {tid }}$ cycles are relatively long with large magnitudes (see Figure 1, type 1 variety seekers). Type 2 consumers $(n=9)$ are distinguished by the fact that they also oscillate about zero by a significant magnitude. In contrast to type 1 , their cycles are more compressed or short in length (see Figure 1, type 2 variety seekers). These consumers desire a very fruity drink at time $t_{1}$, for example, whereas at $t_{3}$ they purchase a drink with very little fruitiness. Type 3 consumers $(n=9)$ are represented by $k_{t i d}$ values which oscillate only about zero by a small amount. These consumers are relatively "value loyal" in that their preferred drinks roughly coincide with their stated ideal level of fruitiness. In addition, their cycles have a long, drawn-out structure (see Figure 1, type 3 variety seekers). Finally, type 4 consumers $(n=7)$ show $k_{t i d}$ values with considerable "ups and downs." Their cycles are small in magnitude, or oscillate close to zero, and are relatively short (see Figure 1, type 4 variety seekers). Type 4 variety seekers are, like type 3 , relatively "value loyal."

The relationship between $k_{\text {tid }}$ and $t$ is estimated by the sine function

$$
k_{t i d}=b_{1} \cdot \sin \left(b_{2} \cdot t+b_{3}\right)+e
$$

where $b_{1}$ describes the magnitude of the oscillation cycle (the extension of the function along the coordinate), $b_{2}$ describes the length of the cycle (the compression of the function along the abscissa where small values of $b_{2}$ reflect longer cycles), $b_{3}$ describes the displacement of the function on the abscissa, and $e$ is the residual. We apply the MarquardtLevenberg algorithm (see Quandt, 1983) to vary the given values of the parameters in an iterative process until the minimum of the objective function is obtained.

\subsection{Results}

Estimation results for a redemption value of .50 are presented at the top of Table 1 . The model $R^{2}$ is 0.53 for type 1 consumers and 0.26 for type 2 . Consistent with Figure 1 , these two consumer types show similar magnitudes in their oscillation cycles ( $b_{1}$ equals 1.630 and 1.781). The estimated length of the cycles is longer for type 1 than for type $2\left(b_{2}\right.$ equals 0.474 versus 1.571 ). In contrast to both type 1 and 2 consumers, a smaller proportion of the total variance is explained for types 3 and $4\left(R^{2}=0.12\right.$ and 0.08 respectively). Compared to types 1 and 2 , consumer types 3 and 4 have estimated oscillation cycles that are smaller in magnitude ( $b_{1}$ equals 0.902 and 0.649 ), which is consistent with the interpretation of these types as relatively "value loyal." Finally, the oscillation cycles are longer for type 3 than for type 4 ( $b_{2}$ equals 0.249 versus 1.178 ).

A number of factors may contribute to the differences in fit across types, including small sample sizes, contextual effects, changes in constraints, the effects of other attributes such as caffeine level, and consumption outside the beverage category. Yet the ability of the model to account for the pattern of consumption across the segments is very promising, especially when one considers that our estimation is based on a single descriptive dimension.

One obvious limitation may be the presumption that the retention factor $(h)$ equals .50 . To examine this, Table 1 also presents model estimation results assuming $h=.25$ and .75 . 
Table 1 . Nonlinear regression results.

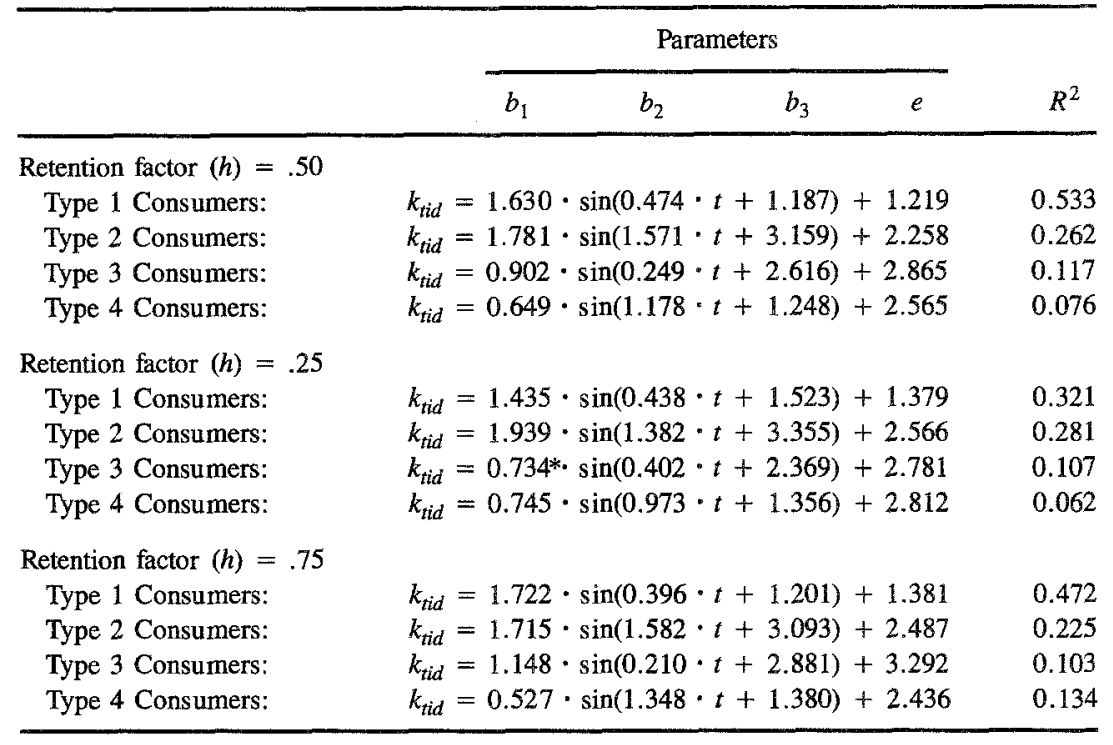

*Significant at $p<.10$. All other parameters are significant at $p<.05$.

Note that the classification of consumers by type and the selection of starting values for the $k_{\text {tid }}$ cycles (described earlier) were relatively robust or stable across the different values of $h$. For comparison purposes, therefore, classifications and starting values obtained for $h=.50$ were used throughout to minimize confounds. Overall, the results are relatively similar across retention factors. A retention factor of .50 provides the best fit for the type 1 consumers. The choice of a retention factor does not have a large effect in the other cases with the exception of type 4 consumers where $R^{2}=0.13$ when $h=.75$ (versus 0.08 when $h=.50$ ). The important point is that, across all three retention factors, the results show a pattern of values for the parameters $b_{1}, b_{2}$, and $b_{3}$, which supports systematic variation about the consumers' ideal points.

\section{Discussion and implications}

While previous balance or satiation models posit that variety seeking is the result of attribute satiation across attributes, the proposed WAVS model predicts systematic variation within the context of an underlying descriptive dimension. Variety seeking among the nested features, or meaningfully different value ranges, of an underlying dimension results in a pattern of consumption on the attribute that may be represented as an oscillation about an ideal point. Conceptually, the model integrates research on satiation-based models of variety seeking with research that describes how attributes are cognitively processed in judgment and choice tasks. Using commonly collected data (perceptions on the dimension, ideal points, and purchase histories), a procedure is described for operationalizing the predicted oscillation patterns and an empirical illustration is described. 
Our empirical illustration identified four types of consumers who varied in the structure of their oscillation patterns. The model does a better job of explaining variety seeking among those consumers whose oscillations show large magnitudes with either longer (type 1) or shorter (type 2) cycles. A nonlinear sine regression function provides a good statistical representation of consumption patterns for these consumers. The model is less able to capture variety seeking among consumers who are more loyal to their ideal point value on the underlying dimension (types 3 and 4) where, in all likelihood, other contextual or external factors explain most of the variance in purchase histories over time.

The estimation procedure is promising in that minimal information is required to operationalize the model and predict the pattern of consumption oscillation for certain consumers. Consumers clearly vary by segment in their oscillation patterns depending on the nature of their preference distribution on an attribute. Foremost, the model stands to improve our ability to predict choice. Knowing where consumers are in their oscillation pattern makes their next choice more apparent than a prediction based on simple base rates. Our findings also underscore why it is important that manufacturers offer a portfolio of beverages that differ with regard to their taste, sugar content, caffeine content, and so on. Such portfolios allow variety seekers to remain faithful to an umbrella brand or manufacturer over time.

One possible path for extending this research is to explicitly model preference levels for each nested feature on a dimension. We expect, for example, that as the preference distribution across features widens, oscillation cycles increase in magnitude. However, even when there is a wide distribution of preferences across the nested features of a dimension, the combination of a low satiation rate and a high decay rate may result in little to no variety seeking.

A second research direction currently being explored is an expansion of the singledimension model to a multidimensional case. Consumers differ, for example, in the degree to which their variety seeking reflects a positive or negative correlation among the attributes in their consideration set. While some consumers may switch between fruity and decaffeinated beverages, others may switch between fruity and caffeinated beverages. Also, the preferred level of an attribute at any given point in time may be a function of both the level of the attribute previously consumed and its interaction with other attributes. Overall it appears that expanded versions of the WAVS model have the potential to explain a wide variety of purchase and consumption behavior.

\section{References}

Bawa, Kapil. (1990). "Modeling Inertia and Variety Seeking Tendencies in Brand Choice Behavior," Marketing Science 3 (Summer), 263-278.

Best, Roger. (1976). "Predictive Aspects of a Joint-Space Theory of Stochastic Choice," Journal of Marketing Research 13 (May), 198-204.

Farquhar, Peter H., and Vithala R. Rao. (1976). "A Balance Model for Evaluating Subsets of Multiattributed Items," Management Science 5 (January), 528-539.

Gati, Itamar, and Amos Tversky. (1982). "Representations on Qualitative and Quantitative Dimensions," Journal of Experimental Psychology: Human Perception and Performance 8(2), 325-340.

Givon, Moshe. (1984). "Variety Seeking through Brand Switching," Marketing Science 3(1), 1-22.

Jeuland, Abel P. (1978). "Brand Preference Over Time: A Partially Deterministic Operationalization of the Notion of Variety Seeking." In Subashi Jain (Ed.), Research Frontiers in Marketing: Dialogues and Directions (Vol. 43), (pp. 33-37). AMA 1978 Educators' Proceedings. Chicago: American Marketing Association. 
Johnson, Michael D., and Claes Fornell. (1987). "The Nature and Methodological Implications of the Cognitive Representation of Products," Journal of Consumer Research 14 (September), 214-228.

Johnson, Michael D., Donald R. Lehmann, Claes Fornell, and Daniel R. Horne. (1992). "Attribute Abstraction, Feature-Dimensionality, and the Scaling of Product Similarities," Intemational Joumal of Research in Marketing 9(1), 131-147.

Lattin, James M. (1987). "A Model of Balanced Choice Behavior," Marketing Science 6 (Winter), 48-65.

Lattin, James M., and Leigh McAlister. (1985). "Using a Variety-Seeking Model to Identify Substitute and Complementary Relationships Among Competing Products," Journal of Marketing Research 22 (August), 330-339.

Lehmann, Donald R. (1972). "Judged Similarity and Brand-Switching Data as Similarity Measures," Journal of Marketing Research 9 (August), 331-334.

McAlister, Leigh. (1982). "A Dynamic Attribute Satiation Model of Variety Seeking Behavior," Joumal of Consumer Research 9 (September), 141-150.

Myers, James H., and Allan D. Shocker. (1981). "The Nature of Product Related Autributes," Research in Marketing 5, 211-236.

Park, C. Whan. (1976). "The Effect of Individual and Situation-Related Factors on Consumer Selection of Judgmental Models," Journal of Marketing Research 13 (May), 144-151.

Park, C. Whan. (1978). "A Conflict Resolution Choice Model," Journal of Consumer Research 5 (September), 124-137.

Prinz, W., and G. Scheerer-Neuman. (1974). "Component Processes in Multiattribute Stimulus Classification," Psychological Research 37(11), 25-50.

Quandt, Richard E. (1983). "Computational Problems and Methods." In Z. Griliches and M. Intriligator (Eds.), Handbook of Econometrics (Vol. 1). Amsterdam: North Holland. 